\title{
Harmine acts as an indirect inhibitor of intracellular protein aggregation
}

Swati Jain, Venkataharsha Panuganti, Sonali Jha and Ipsita Roy*

Department of Biotechnology, National Institute of Pharmaceutical Education and Research, Sector 67, S.A.S. Nagar, Punjab 160062, India

*To whom correspondence to be addressed at Tel: 0091-172-229 2061

Fax: 0091-172-221 4692

Email: ipsita@niper.ac.in 
(a)

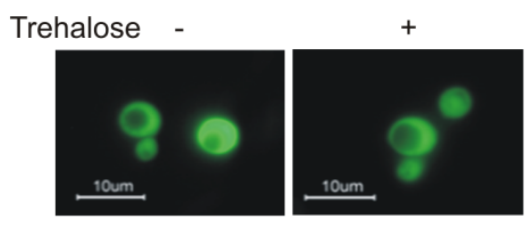

(b)

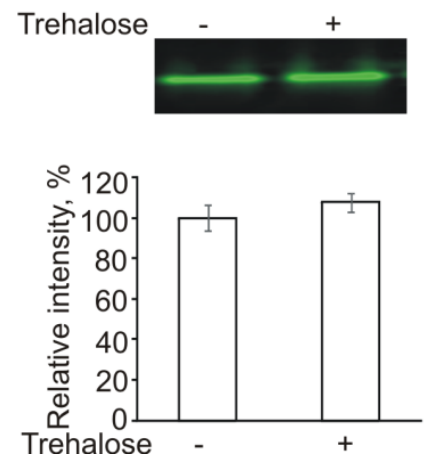

(c)

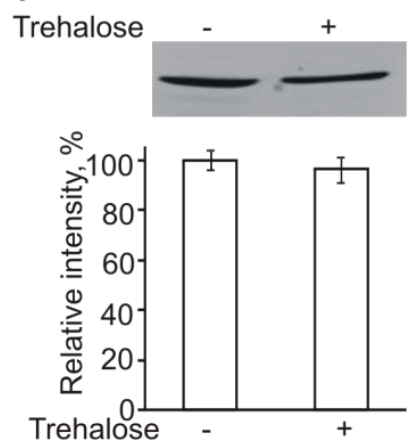

Figure S1. Effect of trehalose on the expression of 25Q-htt. (a) Post-induced yeast cells were pelleted down, washed, mounted on glass slides and viewed under a fluorescence microscope (Nikon E600 Eclipse, Nikon Corporation, Japan). Bar $=10 \mu \mathrm{m}$. (b) Native PAGE analysis of soluble fractions of cell lysates expressing 25Q-htt in the absence and presence of trehalose $\left(4 \%, \mathrm{w} \mathrm{v}^{-1}\right)$. The gel was scanned with an image scanner (Typhoon Trio, GE Healthcare), using $\lambda_{\text {ex }} 532 \mathrm{~nm}, \lambda_{\text {em }} 610 \mathrm{~nm}$. Lower panel shows densitometric analysis of the bands. Band intensity of 25Q-htt in untreated cells (absence of trehalose) was assigned an arbitrary value of $100 \%$. Values shown are mean \pm sem of three independent experiments. (c) Western blotting of soluble fractions of cell lysates expressing 25Q-htt in the absence and presence of trehalose using polyglutamine antibody. Lower panel shows densitometric analysis of the bands. Band intensity of 25Qhtt in untreated cells (absence of trehalose) was assigned an arbitrary value of $100 \%$. Values shown are mean \pm sem of three independent experiments. 


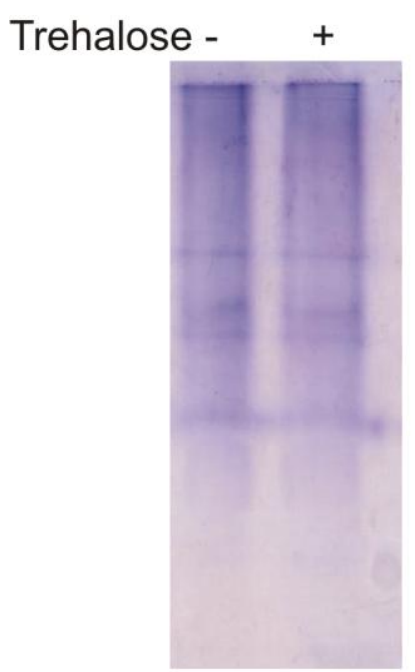

(a)

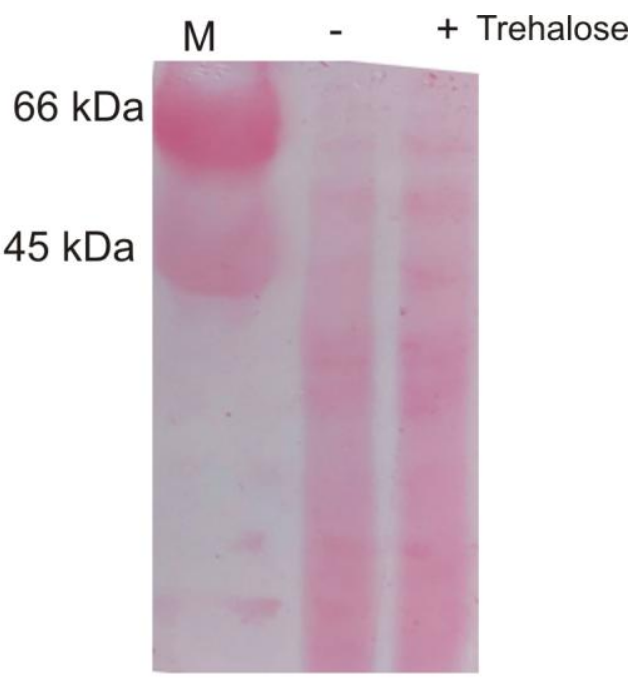

(b)

Figure S2. Trehalose inhibits aggregation of 103Q-htt. (a) Native PAGE analysis of soluble fractions of cell lysates expressing 103Q-htt in the absence and presence of trehalose $\left(4 \%, \mathrm{w} \mathrm{v}^{-1}\right)$. The gel, shown in Figure $1 \mathrm{c}$, was stained with Coomassie following scanning with an image scanner (Typhoon Trio, GE Healthcare). (b) Western blotting of soluble fractions of cell lysates expressing 103Q-htt in the absence and presence of trehalose $\left(4 \%, \mathrm{w} \mathrm{v}^{-1}\right)$. The membrane was stained with Ponceau $\mathrm{S}$ after electrophoretic transfer of protein bands from the polyacrylamide gel to the nitrocellulose membrane. Lane M: Protein markers. The result of probing with antibody is shown in Figure 1d. 


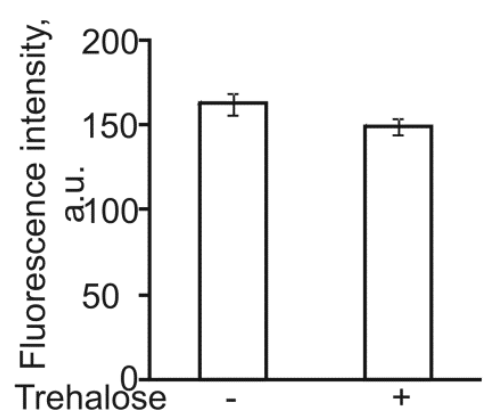

(a)

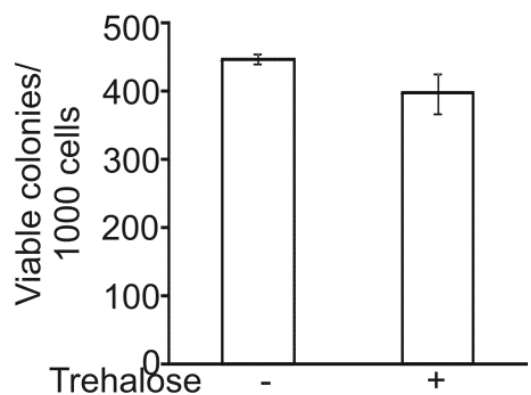

(b)

Figure S3. Trehalose has no effect on oxidative stress and viability of cells expressing 25Q-htt. (a) Estimation of reactive oxygen species (ROS) in cells expressing 25Q-htt in the absence and presence of trehalose using DHE $\left(\lambda_{\text {ex }} 535 \mathrm{~nm}, \lambda_{\text {em }} 635 \mathrm{~nm}\right)$. Values shown are mean \pm sem of three independent experiments. (b) Viability of yeast cells expressing 25Q-htt in the absence and presence of trehalose. Values shown are mean \pm sem of three independent experiments. 
(a)
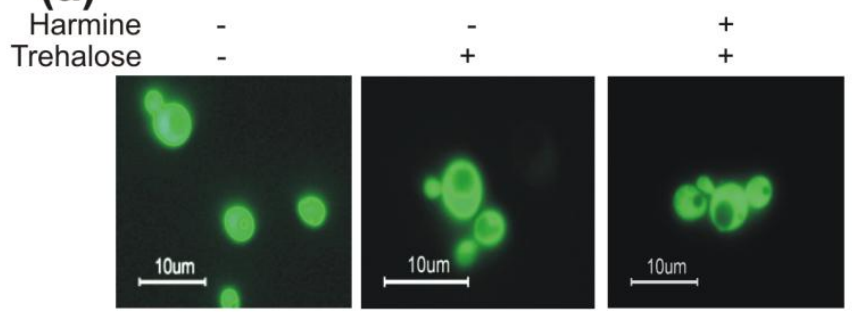

(c)

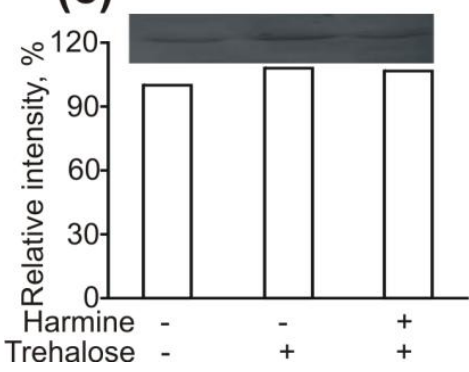

(d)

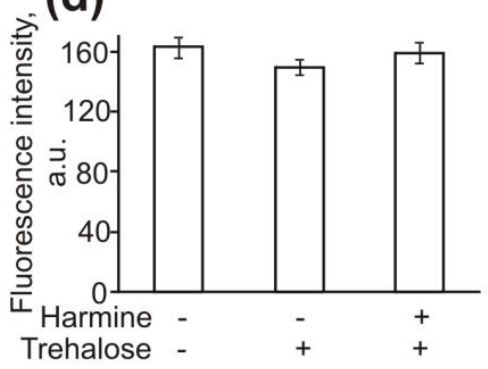

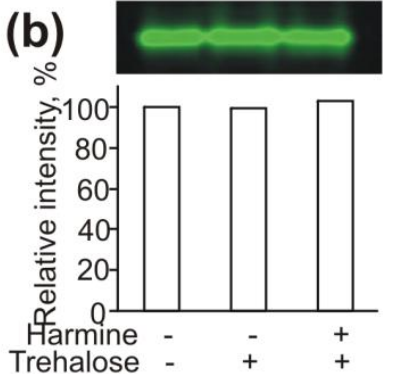

(e)

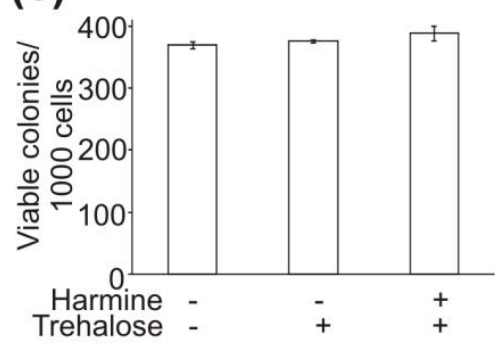

Figure S4. Effect of harmine on the expression of 25Q-htt in yeast cells. (a) Fluorescence images of $S$. cerevisiae cells expressing 25Q-htt in the absence and presence of trehalose $\left(4 \%, \mathrm{w} \mathrm{v}^{-1}\right)$ and harmine $\left(25 \mu \mathrm{g} \mathrm{mL}^{-1}\right)$. Images were visualized under 100X objective and 10X eyepiece. Bar $=10 \mu \mathrm{m}$. Images were taken after $10 \mathrm{~h}$ of induction with galactose and/or trehalose and/or harmine. (b) Native PAGE analysis of soluble fractions of cell lysates expressing 25Q-htt in the absence and presence of trehalose $\left(4 \%, \mathrm{w} \mathrm{v}^{-1}\right)$ and harmine $\left(25 \mu \mathrm{g} \mathrm{mL}^{-1}\right)$. The gel was scanned with an image scanner (Typhoon Trio, GE Healthcare), using $\lambda_{\mathrm{ex}} 532 \mathrm{~nm}, \lambda_{\mathrm{em}} 610 \mathrm{~nm}$. Lower panel shows densitometric analysis of the bands. Band intensity of 25Q-htt in untreated cells (absence of trehalose and harmine) was assigned an arbitrary value of $100 \%$. Values shown are mean \pm sem of three independent experiments. (c) Western blotting of soluble fractions of cell lysates expressing 25Q-htt in the absence and presence of trehalose (4\%, $\mathrm{w} \mathrm{v}^{-1}$ ) and harmine $\left(25 \mu \mathrm{g} \mathrm{mL}^{-1}\right)$ using polyglutamine antibody. Lower panel shows densitometric analysis of the bands. Band intensity of 25Q-htt in untreated cells (absence of trehalose and harmine) was assigned an arbitrary value of $100 \%$. (d) Estimation of reactive oxygen species (ROS) in cells expressing 25Q-htt using DHE $\left(\lambda_{\text {ex }} 535 \mathrm{~nm}, \lambda_{\text {em }}\right.$ $635 \mathrm{~nm}$ ). (e) Viability of yeast cells expressing 25Q-htt in the absence and presence of trehalose $\left(4 \%, \mathrm{w} \mathrm{v}^{-1}\right)$ and harmine $\left(25 \mu \mathrm{g} \mathrm{mL}^{-1}\right)$. Values shown are mean \pm sem of three independent experiments. 


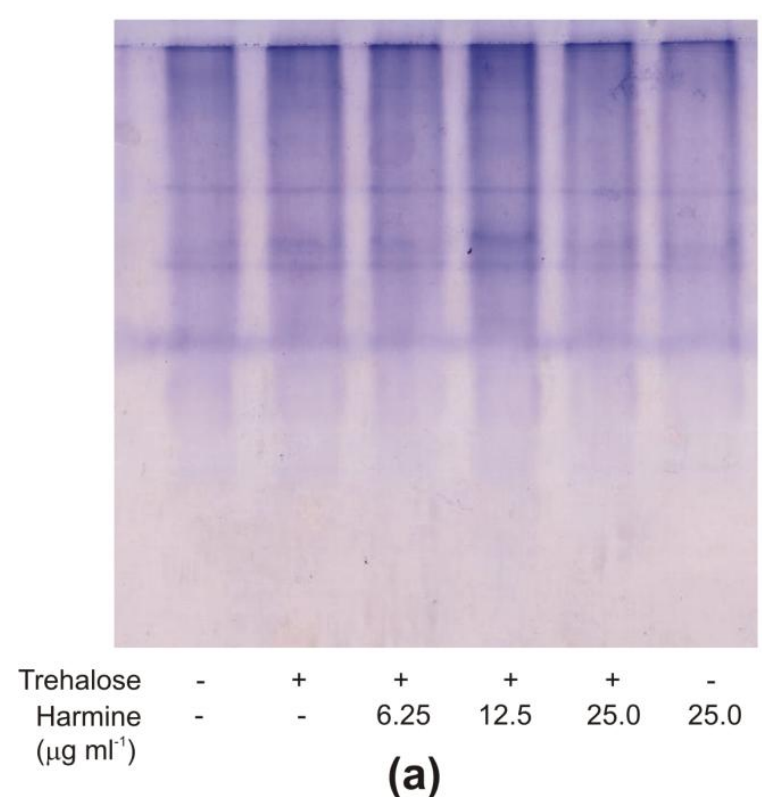

(a)

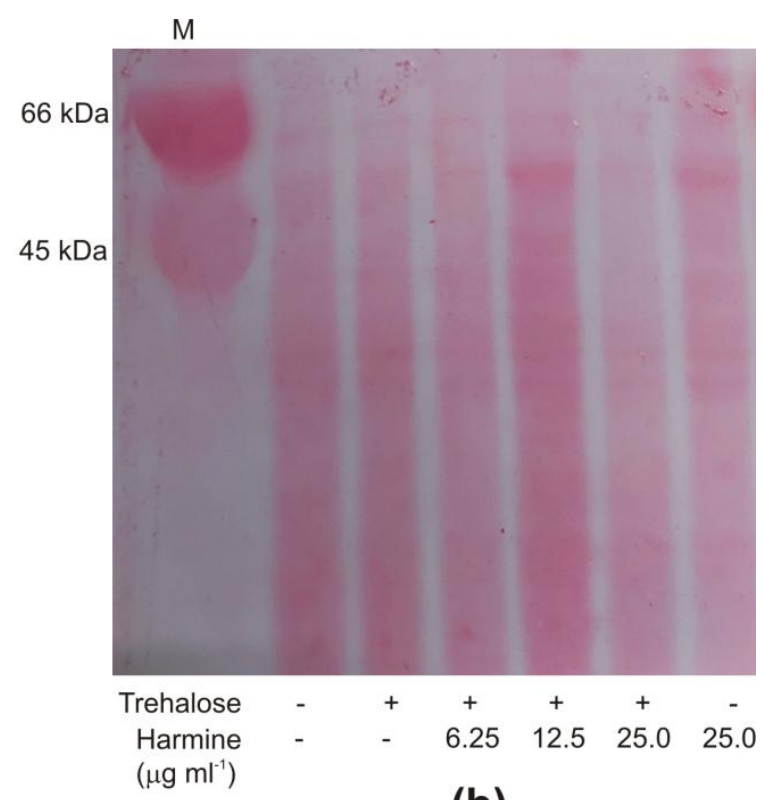

(b)

Figure S5. Harmine attenuates aggregation of 103Q-htt in yeast cells. (a) Native PAGE analysis of soluble fractions of cell lysates expressing 103Q-htt in the absence and presence of trehalose $\left(4 \%, \mathrm{w} \mathrm{v}^{-1}\right)$ and different concentrations of harmine. The gel, shown in Figure 2c, was stained with Coomassie following scanning with an image scanner (Typhoon Trio, GE Healthcare). (b) Western blotting of soluble fractions of cell lysates expressing 103Q-htt in the absence and presence of trehalose $\left(4 \%, \mathrm{w} \mathrm{v}^{-1}\right)$ and different concentrations of harmine. The membrane was stained with Ponceau $\mathrm{S}$ after electrophoretic transfer of protein bands from the polyacrylamide gel to the nitrocellulose membrane. The result of probing with antibody is shown in Figure 2d. Lane M: Protein markers. 


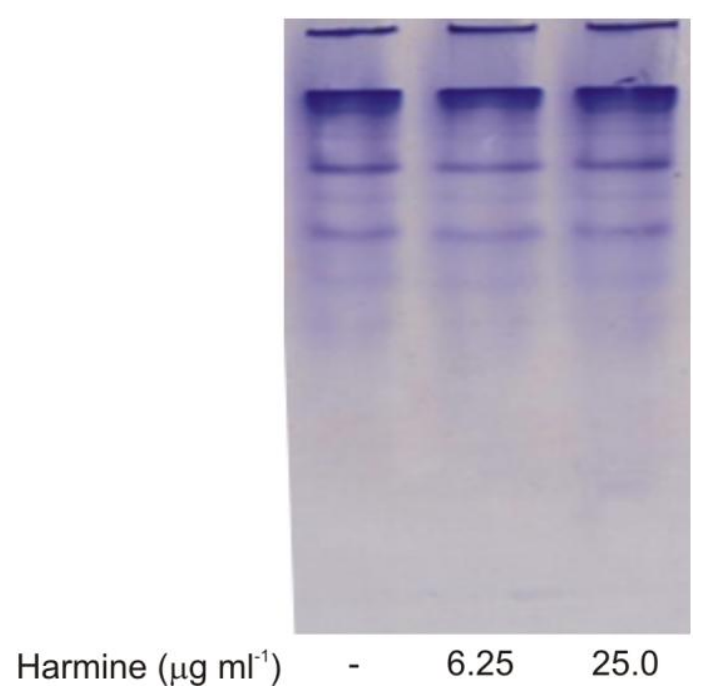

Figure S6. Harmine attenuates prion formation by Rnq1 in yeast cells. Native PAGE analysis of soluble fractions of cell lysates overexpressing Rnq1-EGFP in the absence and presence of harmine. The gel, shown in Figure 2h, was stained with Coomassie following scanning with an image scanner (Typhoon Trio, GE Healthcare). 


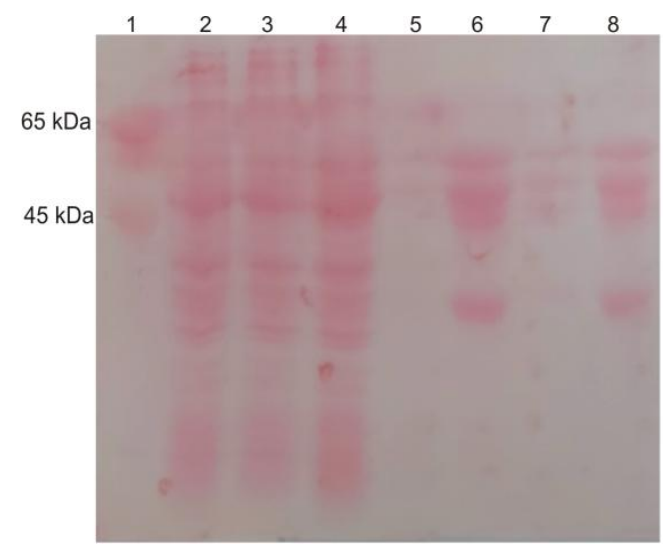

Figure S7. Western blot analysis of purification of GST-51Q-htt. The membrane was stained with Ponceau $S$ after electrophoretic transfer of protein bands from the polyacrylamide gel to the nitrocellulose membrane. The result of probing with antibody is shown in Figure 3b. Lane 1: protein markers, Lane 2: cell lysate, Lane 3: flowthrough, Lanes 4 and 5: washings, Lanes 6 and 7: eluted GST-51Q-htt, Lane 8: dialyzed protein. Protein load was $20 \mu \mathrm{g}$ each lane. 
(a)

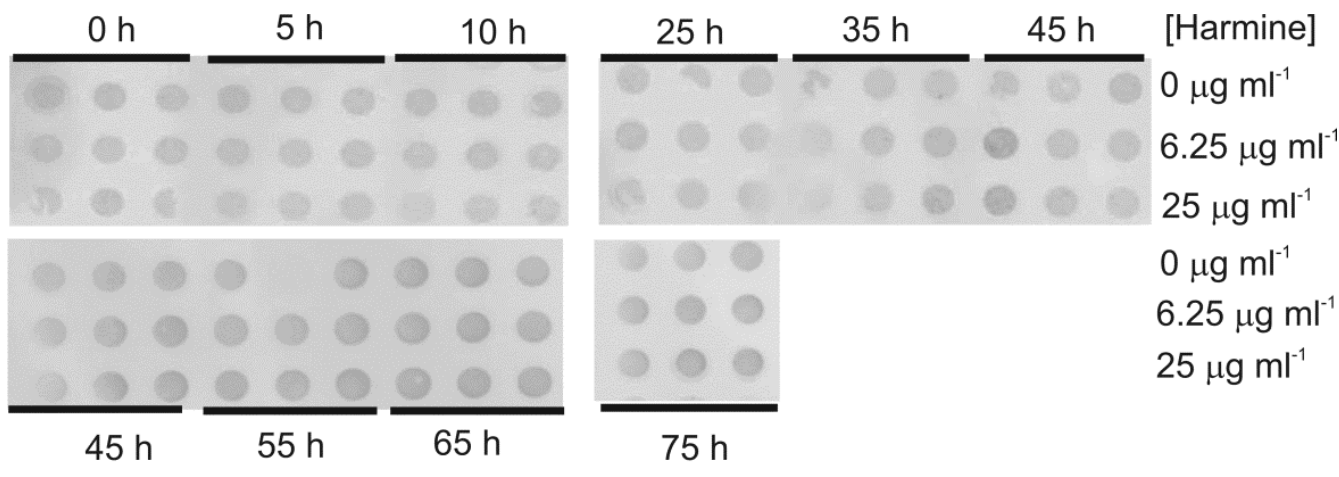

(b)
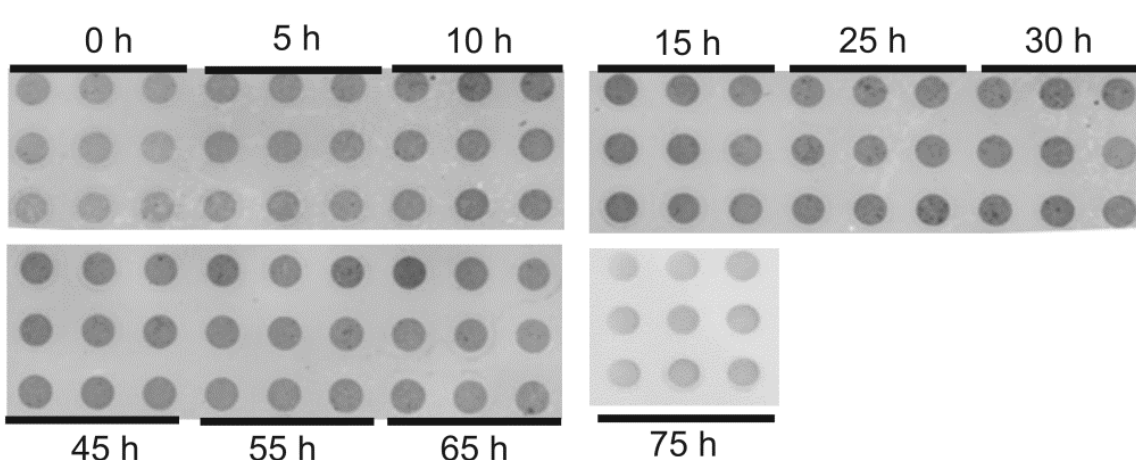

Harmine]

$0 \mu \mathrm{g} \mathrm{ml}^{-1}$

$6.25 \mu \mathrm{g} \mathrm{ml}^{-1}$

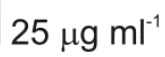

$0 \mu \mathrm{g} \mathrm{ml}^{-1}$

$6.25 \mathrm{\mu g} \mathrm{ml}^{-1}$

$25 \mu \mathrm{g} \mathrm{ml}^{-1}$

Figure S8. Effect of harmine on aggregation pattern of 51Q-htt monitored by (a) filter retardation assay using the polyglutamine antibody and (b) dot-blot assay using the oligomer-specific A11 antibody. Triplicate dots are shown for each time point. 